\title{
Radiation-Hard/High-Speed Parallel Optical Links
}

\author{
K.K. Gan', H. Kagan, R.D. Kass, J. Moore, D.S. Smith, \\ Department of Physics, \\ The Ohio State University, \\ Columbus, $\mathrm{OH} 43210$, USA \\ E-mail: ganemps.ohio-state.edu
}

P. Buchholz, A. Wiese, and M. Ziolkowski

Fachbereich Physik,

Universität Siegen,

Siegen, Germany

E-mail: Michael.Ziolkowski@uni-siegen.de

We have designed an ASIC for use in a parallel optical engine for a new layer of the ATLAS pixel detector in the initial phase of the LHC luminosity upgrade. The ASIC is a 12-channel VCSEL (Vertical Cavity Surface Emitting Laser) array driver capable of operating up to $5 \mathrm{~Gb} / \mathrm{s}$ per channel. The ASIC is designed using a $130 \mathrm{~nm}$ CMOS process to enhance the radiationhardness. A scheme for redundancy has also been implemented to allow bypassing of a broken VCSEL. The ASIC also contains a power-on reset circuit that sets the ASIC to a default configuration with no signal steering. In addition, the bias and modulation currents of the individual channels are programmable. We have received the ASIC and the performance up to $5 \mathrm{~Gb} / \mathrm{s}$ is satisfactory. Furthermore, we are able to program the bias and modulation currents and to bypass a broken VCSEL channel. We briefly present our plan to upgrade the design to allow operation at $10 \mathrm{~Gb} / \mathrm{s}$ per channel yielding an aggregated bandwidth of $120 \mathrm{~Gb} / \mathrm{s}$.

36th International Conference on High Energy Physics

July 4-11, 2012

Melbourne, Australia

${ }^{1}$ Speaker 


\section{Introduction}

A parallel optical engine allows a compact design for high-speed data transmission. The design is enabled by readily available high-speed VCSEL arrays. With the use of a 12-channel array operating at $10 \mathrm{~Gb} / \mathrm{s}$ per channel, a parallel optical engine can deliver an aggregate bandwidth of $120 \mathrm{~Gb} / \mathrm{s}$. With a standard spacing of $250 \mu \mathrm{m}$ between VCSELs, the width of a 12-channel array is only slightly over $3 \mathrm{~mm}$. This allows the fabrication of a rather compact parallel optical engine for installation in locations where space is at a premium. Besides the reduced physical size, there are other advantages in using an array solution. For example, one can reserve 1 in 12 channels for redundancy instead of doubling the number of channels if using a single-channel VCSEL. This is therefore a much more efficient scheme to implement redundancy for bypassing a dead channel. The array scheme also reduces the service requirements by using one power supply to bias each array instead of a single power supply for each VCSEL channel. Additionally, the use of a fiber ribbon reduces the number of fibers to handle and moreover a fiber ribbon is less fragile than a single-channel fiber. These multiple advantages greatly simplify the production, testing, and installation of optical links.

VCSEL arrays are widely used in off-detector data transmission in high-energy physics. The first implementation of VCSEL arrays for on-detector application is in the optical links of the ATLAS pixel detector. The experience from the operation has been quite positive despite failures in the optical readout system [1]. Modern VCSELs are humidity tolerant and hence no special precautions are needed. The ATLAS experiment plans to continue the use of VCSEL arrays in a new layer of the pixel detector, the insertable barrel layer (IBL), to be installed in the initial phase of the LHC luminosity upgrade.

In response, we have designed an ASIC for possible use in the IBL project. The ASIC is a 12-channel VCSEL array driver capable of operating up to $5 \mathrm{~Gb} / \mathrm{s}$ per channel. We will present some measurements of the characteristics of the ASIC below. We have also modified the design for operation at $10 \mathrm{~Gb} / \mathrm{s}$ per channel and some preliminary design will be presented.

\section{Results from a $5 \mathrm{~Gb} / \mathrm{s}$ VCSEL array driver}

The VCSEL array driver ASIC is designed to operate at speed up to $5 \mathrm{~Gb} / \mathrm{s}$. The planned operating speed for the IBL optical link is $160 \mathrm{Mb} / \mathrm{s}$ but we designed the ASIC to operate at much higher speed to gain experience in the design of a high-speed array driver. The ASIC was fabricated in a $130 \mathrm{~nm}$ CMOS process to enhance the radiation-hardness [2]. All circuitry was designed following test results and guidelines from CERN on radiation tolerant design in the $130 \mathrm{~nm}$ process used [3]. The ASIC is an improved version of the driver designed with a 250 nm CMOS process for use in the optical links [4] of the installed pixel detector of the ATLAS experiment. Several improvements were incorporated into the new ASIC taking advantage of experience gained from building and operating the first array-based optical links and will be detailed below.

Each ASIC contains eight low voltage differential signal (LVDS) receivers to receive the data from the front-end electronics. A block diagram of the ASIC is shown in Figure 1. The received LVDS signal is converted in the driver stage into a current sufficient to drive a 
VCSEL. The amplitude of the modulation current in each driver is controlled via an 8-bit DAC. There is also a single 8-bit DAC to set the bias currents of all of the channels simultaneously. In addition, there are four spare drivers with associated 8-bit DACs that can receive the LVDS signal from any of the eight LVDS receivers via a switching network. This allows the received LVDS signal to be transmitted via one of the four spare VCSEL channels should a VCSEL in one of inner eight channels become non-operational. To enable operation in case of a failure in the communication link to the receiver array ASIC, we have included a power on reset circuit that will set the modulation current in the VCSEL to $9 \mathrm{~mA}$ on top of a bias current of $1 \mathrm{~mA}$ upon power up with no signal steering.

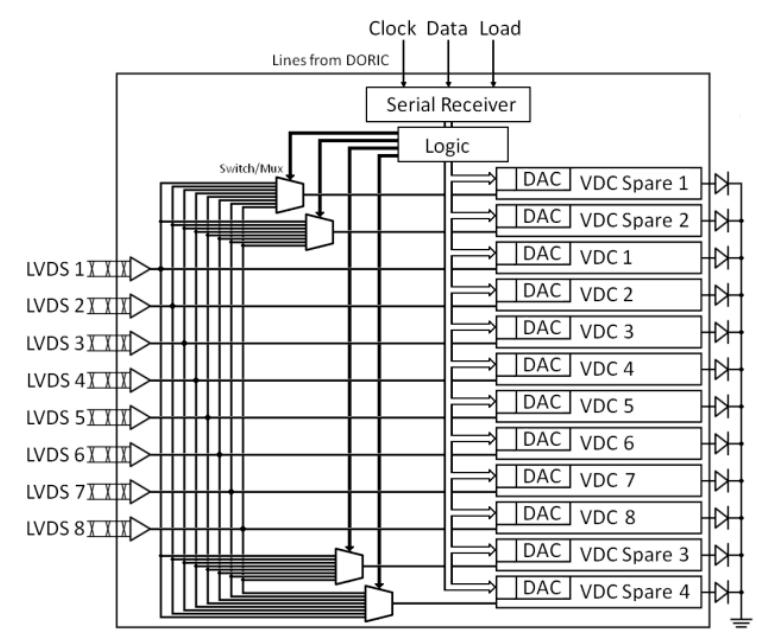

Figure 1: A 12-channel VCSEL array driver ASIC.

The 8-bit DAC and LVDS receiver are designed to operate with a $1.5 \mathrm{~V}$ supply and the driver stage is designed to operate with a $2.5 \mathrm{~V}$ power supply. A higher supply voltage is required by the output stage to allow enough headroom to drive VCSELs which normally have threshold voltages of $2 \mathrm{~V}$ or higher.

We have characterized several ASICs. Each ASIC is coupled to a VCSEL array fabricated by ULM with a bandwidth of $10 \mathrm{~Gb} / \mathrm{s}$ [5]. The current consumption for the $2.5 \mathrm{~V}$ supply is measured to $250 \mathrm{~mA}$ with all channels active at $5 \mathrm{~Gb} / \mathrm{s}$. The power consumption for the $1.5 \mathrm{~V}$ supply is $30 \mathrm{~mA}$. The ASIC can supply at least $10 \mathrm{~mA}$ of drive current to a VCSEL, producing an optical power in excess of $1 \mathrm{~mW}$.

The optical eye diagram of a channel in the driver ASIC is shown in Figure 2. All other channels in the ASIC were active with the same pseudo-random input signal but delays introduced by cabling and buffer chips distributing the signals effectively desynchronize the signals into each VCSEL driver channel. It is evident that the eye is open but the test is limited by the bandwidth of the $4.5 \mathrm{GHz}$ optical probe available in our lab.

Due to the limitations of the optical probe described above, we evaluate the performance of the driver ASIC using a Finisar Small Form Factor (SFP+) transceiver. This SFP+ transceiver is specified to operate at $10 \mathrm{~Gb} / \mathrm{s}$ and thus we can use it to probe of the quality of the received the optical signal from the VCSEL. This is accomplished by feeding the SFP+ electrical output to a $13 \mathrm{GHz}$ oscilloscope. It should be noted that the use of the SFP+ transceiver improves the 
vertical eye opening. The transceiver discriminates the optical signal and thus removes amplitude noise by re-shaping the wave fronts. Figure 3 shows the eye diagram obtained with the $\mathrm{SFP}+$ transceiver. It is evident that the eye diagram readily satisfies the IEEE specification for the received electrical eye with a clear separation between the signal traces and the mask [6]. The cause of the double band structure is still under investigation.

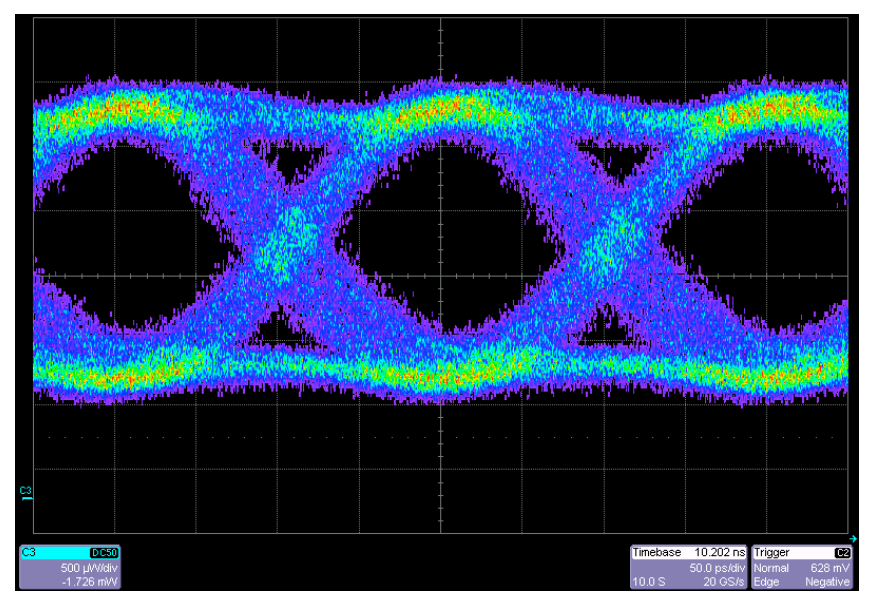

Figure 2: Optical eye diagram of a VCSEL channel coupled to a driver ASIC.

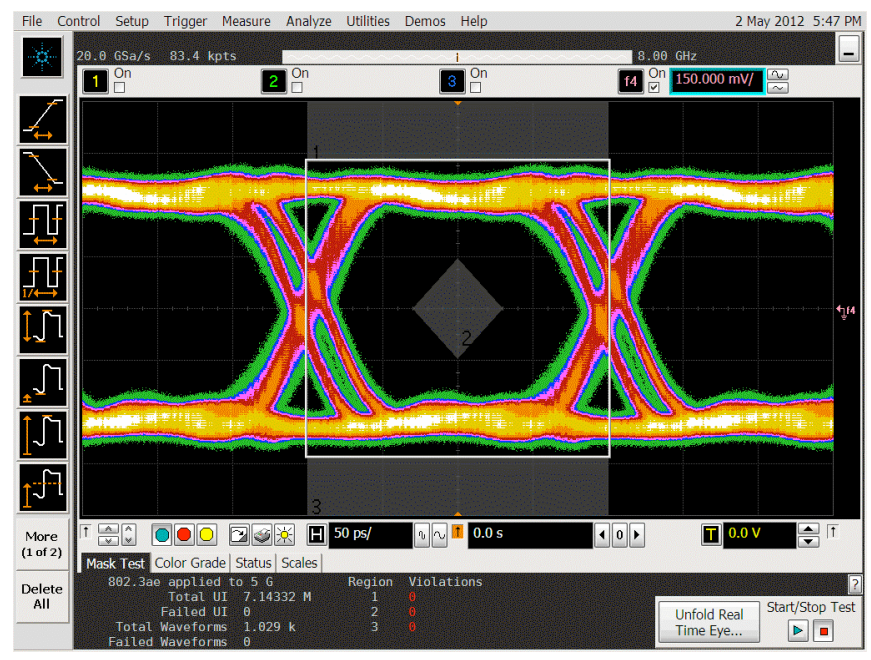

Figure 3: Eye diagram of a VCSEL channel coupled to a driver ASIC as viewed with a SFP+ transceiver. Only one driver channel is active. The IEEE mask for $5 \mathrm{~Gb} / \mathrm{s}$ transmission is indicated.

The above eye diagram is for the case in which only one VCSEL driver channel is active. Figure 4 shows the eye diagram with all channels active, a more realistic operating condition. It is evident that the jitter is somewhat larger but that the eye diagram still readily satisfies the IEEE specification. With all channels active at $5 \mathrm{~Gb} / \mathrm{s}$, the bit error rate is less than $5 \times 10^{-13}$ and the total jitter is $65 \mathrm{ps}$ at the bit error rate of $5 \times 10^{-12}$, including the contribution of $3.1 \mathrm{ps}$ from random jitter and $36.8 \mathrm{ps}$ from deterministic jitter. 


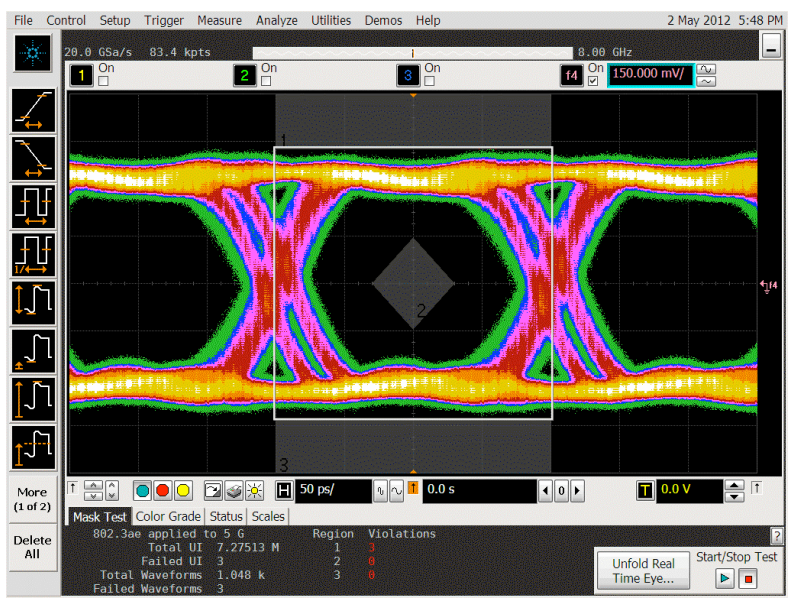

Figure 4: Eye diagram of a VCSEL channel coupled to a driver ASIC as viewed via a SFP+ transceiver. All driver channels are active. The IEEE mask for $5 \mathrm{~Gb} / \mathrm{s}$ transmission is indicated.

As noted above, the ASIC also contains several added functionalities to make the optical links more reliable and easier to operate, motivated by the experience in operating the first ondetector optical links based on VCSEL arrays. This includes a power-on reset circuit that sets the ASIC to a default configuration with no signal steering in case of a failure in the communication link to the ASIC. This feature has been successfully implemented. We also verified that the bias and modulation currents of the individual channels are programmable. The power-on reset circuit sets the ASIC to produce $9 \mathrm{~mA}$ of modulation current and $1 \mathrm{~mA}$ of bias current. This has also been verified.

The redundancy circuit is an important feature implemented for the bypassing of a broken VCSEL. The integrity of the steered signal in the spare channel has been tested by steering the signal through the maximum distance from a channel on one side of the ASIC to the spare channel on the other side. Figure 5 shows the eye diagram of this signal. The steered signal has more jitter as expected but the eye diagram still satisfies the IEEE specification with large margin.

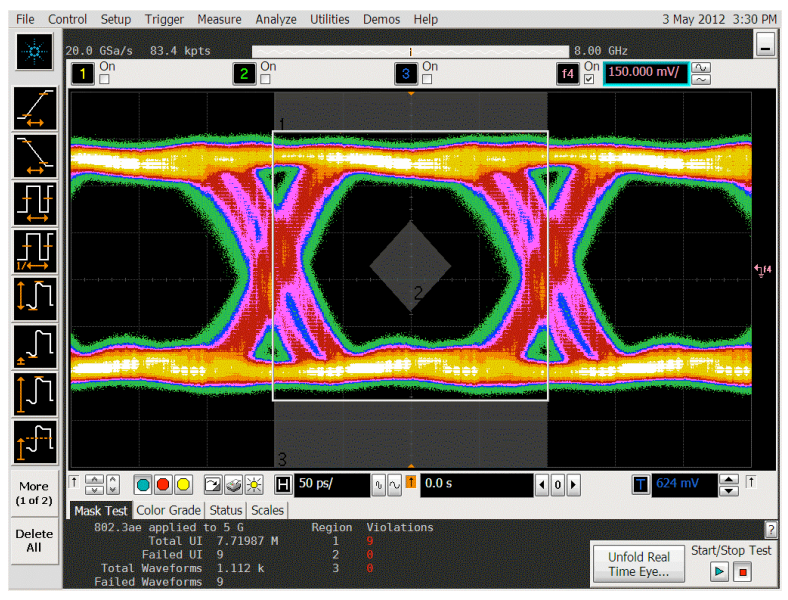

Figure 5: Eye diagram of the steered signal in a spare channel as viewed via a SFP+ transceiver. All driver channels are active. The IEEE mask for $5 \mathrm{~Gb} / \mathrm{s}$ transmission is indicated. 


\section{Future plan}

We are currently designing a $10 \mathrm{~Gb} / \mathrm{s}$ VCSEL driver to yield an aggregated bandwidth of $120 \mathrm{~Gb} / \mathrm{s}$ when coupled to a 12-channel VCSEL array. A central question of the design is what semiconductor process to use. We expect both $130 \mathrm{~nm}$ SiGe BiCMOS and $65 \mathrm{~nm}$ CMOS to have the sufficient bandwidth. However, due to cost consideration, we will first attempt to design the driver using the $130 \mathrm{~nm}$ CMOS process to see if this is practical.

\section{Conclusions}

We present the result of a $5 \mathrm{~Gb} / \mathrm{s}$ VCSEL array driver ASIC for use in a parallel optical engine. The ASIC is designed using a $130 \mathrm{~nm}$ CMOS process to enhance the radiation-hardness. The eye diagram is open, satisfying the IEEE specification with good margin. The added functionalities to make the optical engine more reliable and easier to operate are also successful. This includes the ability to set modulation current for each VCSEL individually and the bias current for the whole array. Also included is a redundancy circuit to bypass a broken channel and a power-on reset circuit to set the ASIC to a default configuration with no signal steering and a default bias and modulation currents in a VCSEL. For the future, we plan to upgrade the design to operate at $10 \mathrm{~Gb} / \mathrm{s}$ for use in a parallel optical engine to produce an aggregated bandwidth of $120 \mathrm{~Gb} / \mathrm{s}$.

\section{Acknowledgments}

This work was supported in part by the U.S. Department of Energy under contract No. DE-FG-02-91ER-40690 and by the German Federal Minister for Research and Technology (BMBF) under contract No. 056Si74.

\section{References}

[1] Mark S. Cooke, in Proceedings of DPF-2011, Providence, RI, August 8-13, 2011 [hepex/1109.6679v1].

[2] L. Gonella et al., Nucl. Instrum. Methods. A 582 (2007) 750.

[3] F. Faccio, personal communication, 2007.

[4] K. Arms et al., ATLAS pixel opto-electronics, Nucl. Instrum. Methods. A. 554 (2005) 458.

[5] The VCSEL array used is ULM850-10-TN-N0112U, fabricated by ULM Photonics.

[6] The SFP+ transceiver used is FTLX8571D3BCL, fabricated by Finisar.

[7] The mask was adapted for $5 \mathrm{~Gb} / \mathrm{s}$ transmission from the IEEE $802.3 \mathrm{ae}$ specification for $10 \mathrm{~Gb} / \mathrm{s}$ links. 